Benno Engels/Ullrich LaAser (Hrsg.):

Deutsche Bildungshilfe in der zweiten Entwicklungsdekade.

Eine Zwischenbilanz, Weltforum Verlag München 1978, 569 S., 54 DM

Bei dem vorliegenden Reader handelt es sich um die Aufbereitung von Materialien und Entwicklungsländererfahrungen zu einem Bereich, über den bislang kaum etwas aus dem Dschungel der daran beteiligten Organisationen (wie z. B. das BMZ, die Kulturabteilung des Auswärtigen Amtes, weitere Ministerien, die Bundesländer, politische Stiftungen, Kirchen, Entwicklungsdienste etc.) an die Offentlichkeit gedrungen ist.

Uber das hinausgehend, was der Titel verspricht, enthält der Band auch einige Beiträge zum internationalen bzw. Bildungstransfer der westlichen Industrieländer. Neben einigen, die deutsche Bildungshilfe und -forschung mehr generell analysierenden Beiträgen bietet der Reader ein interessantes Spektrum von bildungspolitischen Fallbeispielen aus der Dritten Welt, an denen deutsche Bildungsforscher und -experten in der einen oder anderen Weise beteiligt sind (waren). Bedauerlich ist, daß der Reader, von wenigen Ausnahmen abgesehen, überwiegend den Eindruck vermittelt, als ob Interessenkonflikte zwischen Nord und Süd und fortdauernd Prozesse der Unterentwicklung für die Bildungshilfe nicht existent (oder totschweigbar) wären, so daß ein überzeugender Begründungszusammenhang für die bisher recht mageren vorzeigbaren Resultatefehlt. Zu größeren Hoffnungen berechtigen immerhin zwei Bildungsaktionen (in Tanzania und Benin), die weitgehend von Selbsthilfeanstrengungen der Bevölkerung getragen waren - eine Voraussetzung, die richtungweisend der deutschen Bildungshilfe in der dritten Entwicklungsdekade zugrunde gelegt werden sollte.

Renate Nestvogel

\title{
Völkerrechtliche Verträge
}

Textausgabe. Herausgegeben von Friedrich Berber in Gemeinschaft mit Albrecht Randelzhofer

2. Auflage, 1979, Deutscher Taschenbuchverlag (Beck-Texte), dtv Nr. 5031, 476 S.,

DM 12,80

\section{Michael Schweitzer / Walter Rudolf (Hrsg.)}

\section{Friedensvölkerrecht}

2. Auflage, 1979, Nomos Verlagsgesellschaft, Baden-Baden, 600 S., DM 24,80

Die Vorauflage des dtv-Bändchens, 1973 erschienen, hatte sich bereits als unentbehrliches Handwerkszeug etabliert; die Herausgeber haben es nun auf den Stand vom 1. Juni 1979 gebracht. Neu aufgenommen wurden die beiden Menschenrechtspakte vom 17. Dezember 1966, die zwischenzeitlich für die Bundesrepublik Deutschland in Kraft getreten sind, und die beiden Zusatzprotokolle von 1977 zu den Genfer Rotkreuzabkommen. Die Beschränkung auf universelles und ,,europäisches“ Völkerrecht führt dazu, daß unter ,,Internationale Organisationen" nur die Satzungen der Vereinten Nationen und des Europarates abgedruckt sind. Auch ist das Recht der Europäischen Gemeinschaften weiterhin ausgeklammert. Soweit die verbindlichen Texte der Verträge nicht in deutscher Sprache formuliert sind, bringt die Sammlung amtliche, offiziöse oder eigene Ubersetzungen. Umfangreicher, aber nicht weniger handlich als der Beck-Text ist das Taschenbuch von Schweitzer und Rudolf, das sich in die Hauptabschnitte ,,Mensch im Völkerrecht“", ,Raum im Völkerrecht“, „Zwischenstaatliche Beziehungen“, ,Internationale Organisationen“ gliedert. Nicht aufgenommen sind, wie schon der Titel des Bandes zeigt, kriegsrechtliche Normen (wohl aber 